\title{
THE THERMAL RADIATION IMPACT OF A CURRENT CARRYING CONDUCTOR AND A METALLIC OBJECT: A CASE STUDY OF TRANSFORMER G\& P ACCESSORIES AND ALUMINUM LADDER
}

\author{
Okoye I. F. (NIP, NAMP, SAN, NATE). \\ Department of Physics, Federal University Gusau, Zamfara State Nigeria.
}

\begin{abstract}
The thermal radiation impact of a current carrying conductor and a metallic object cannot be neglected. The improper knowledge of this effect and the causes are one of the major reasons that contribute to the high number of electrocution cases registered in Nigeria at various levels. This research reveals the neglected magnetic effect that exist around a current carrying conductor and a metallic object near it as one of the silent causes of electric hazard that kill people on daily basis. The researcher observered that electrons are readily available on Aluminum ladder and that photon energy from sunlight or thermal radiation usually powers this electron on the metallic ladder without the knowledge of the operator thereby causing current to start flowing on both the ladder and the current carrying conductor in the same direction. This phenomenon causes current to start flowing on the two naked conductors without the knowledge of some operators who thought that current is only flowing on transformer accessories ( $\mathrm{G}$ and $\mathrm{P}$ unit). The force of attraction acts on two conductors carrying current in the same direction. For this reason, the current from photon energy (thermal radiation) and electrons flow spontaneously in all directions thereby attracting the current that flows from transformer accessories ( $\mathrm{G}$ and $\mathrm{P}$ unit) placed 5 meters distance from the Aluminum ladder. The magnetic impact of this explanation is very deadly as it could result to what is known as electrocution. The researcher recommended the possible way of reducing electrical hazard that might arise from such incidence. Thermal radiation can lead to "Switching -ON" of a device to supply current when the majorities of the number of holes are equals to the no of electrons. Thermal radiation can equally leads to "Switching Off" of a device to stop supplying current when the no of holes is less than number of electrons. Naturally, every semiconductor material has a good reasonable no of electrons existing on the surface of that material and the sunlight is a good carrier of photon.
\end{abstract}

Keywords: Thermal radiation, Magnetic impact, conductor, electrocution, Photon energy and transformer accessories, semiconductor material.

DOI: $10.7176 /$ APTA/80-07

Publication date:October $31^{\text {st }} 2019$

\subsection{INTRODUCTION}

Thermal radiation are synonymous with semiconductor materials (Williams,1992). Semiconductor materials are good carrier of electric current. Semiconductor material can serve as a magnet, switch, conductor etc. Semiconductor elements are found in group III and group V in the table of element. Element like As, Sb or P exist in group V while in group III element like B, Al, Ga or In are found. Some of these element have more electrons than holes while some have more holes than electrons (Williams,1992). The two elements need to be balance in a material for steady flow of current to be established. Also sunshine or thermal radiation energy generates its own photon energy through electromagnetic space to the earth (Okoye and Maduka, 2015). Some dwellers doesn't have proper knowledge of radiant energy from sunlight. Energy from sun light can switch -on or power electron found in a semiconductor material with lesser photon. This idea is one hidden factor that contributes to high number of electricity hazard recorded every day. Thermal radiation is a mandatory event that took place in every metal that is exposed to sunlight or subjected to passage of current or heat (Okoye, 2017). Thermal radiation can lead to "Switching-ON" of a device to supply current when the majorities of the number of holes are equals to the no of electrons. Thermal radiation can equally leads to "Switching Off" of a device to stop supplying current when the no of holes is less than number of electrons.

Naturally, every semiconductor material has a good reasonable no of electrons existing on the surface of that material and the sunlight is a carrier of photon. Once semiconductor material is exposed to sunlight, the metal in question can receive photon energy which will then power the metal and cause current to start flowing in a particular direction (Okoye, 2017). Assuming that the current from a metallic object (Aluminum Ladder) is placed 
within 5 meters range from another conductor carrying current like transformer accessories ( $G$ and $P$ unit), magnetic influence existing between the two conductor carrying current in the same direction will attract or magnet each other. This action is very dangerous because many dwellers don't have knowledge of this idea. That is why many people end up becoming victim when they place their hands or legs on a metallic object placed on conductor carrying electricity in the same direction to the flow of current with the metallic object. Because of this reason, the distance between two conductor carrying current in the same direction to each other must be above 5 meters range to neutralize the magnetic influence that exist between the two conductors.

\subsection{THERMAL RADIATION}

Transformer accessories ( $\mathrm{G}$ and $\mathrm{P}$ unit) and aluminium ladder are all bodies that emit thermal radiation on their own or absorb or reflects radiation incident on them. All bodies emit thermal radiation (Anene and Ekpunobi,1997). All bodies are either grey or black. Grey body either absorbs or reflects radiation incident upon it while a blackbody is defined as a body which absorbs the entire radiation incident upon it. Bodies which are hot enough emit Radio waves, X-rays and gamma rays and travel very much faster at $300,000 \mathrm{Km}$ per second. Extremely hot bodies such as stars emit Radio waves, X-rays and gamma rays and travel with the speed of light (Anene and Ekpunobi, 1997). Black body radiation depends on four things:

1. The frequency of the incident radiation

2. The direction of arrival of incident radiation relative to the body surface.

3. The physical state of the body (Gas, Liquid, Solid).

4. The temperature of the body.

\subsubsection{Perfect Blackbody:}

This is a body that absorbs all the electromagnetic waves falling on it (Halliday etal, 2001). Generally, all objects can emit and also absorb electromagnetic waves. So when an object is in thermal equilibrium with its surroundings, it implies that the amount of radiant energy the object absorbs balances with the amount the object emits. However, if the absorption is greater than the emission, the object will experience a net gain of radiant energy and thus the temperature will rise. If the emission is greater than the absorption, the object will experience a net loss of radiant energy and the temperature will fall. A good absorber is also a good emitter while a poor absorber is also a poor emitter (Francis etal, 1975). Hence, a perfect black body is a perfect absorber and also a perfect emitter of radiant energy.

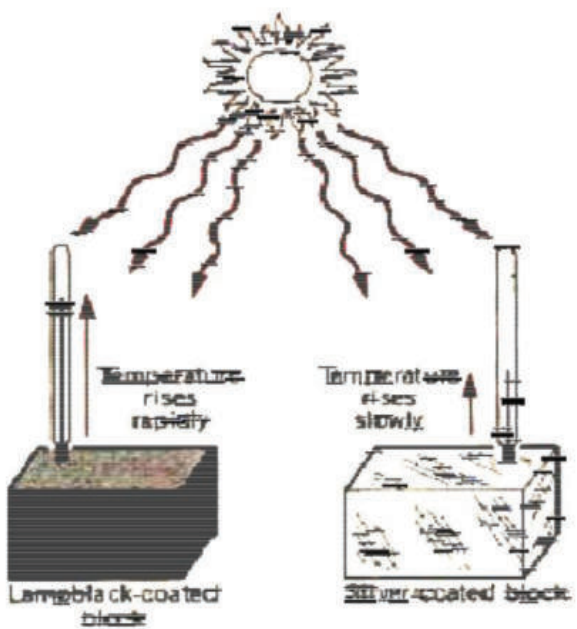

Fig. 1.0: System that shows Perfect Blackbody :(After Cutnell and Johnson, 1989)

\subsection{ELECTROMAGNETIC WAVES AND PHOTONS}

Light is an electromagnetic wave composed of both electric and magnetic fields (Jeff, 1993).

Light waves are called transverse waves because they oscillate in a direction transverse (perpendicular) to the direction in which electric field and magnetic fields travel (Jeff, 1993). Light and all other electromagnetic radiation are forms of energy. The higher the frequency or shorter the wavelength, the more energy the photon 
carries. The powered transformer accessories always have higher frequency or shorter wavelength which is capable of generating more energy through the photon carriers.

Matter absorbs and emits photons. When it absorbs a photon it gains energy; when it emits or radiates a photon, it release energy. The amount of energy can be increased by increasing the photons energy, increasing the number of photons, or both.

If the electron gets too much energy, it escapes from the atom altogether, a process called ionization. Energy of ionized hydrogen atom at zero level is given as;

$\mathrm{E}=-\mathrm{R} / \mathrm{n}^{2}$

Where $\mathrm{R}$ is a constant $\left(2.179 \times 10^{-18} \mathrm{~J}\right)$ and $\mathrm{n}$ is the quantum number of the orbit (counting outwards, with 1 the innermost levels).

\subsection{METHODS OF HEAT TRANSFER}

Heat is a form of energy that can be transferred from one body to another. The transfer of heat from one part of a system to another or to another system by virtue of a temperature difference can only be by one or more of the three processes namely; conduction, convection, and radiation(Francis etal, 1975).

Heat can be transferred by three (3) main mechanisms namely; Convection, Radiation and Conduction.

\subsubsection{Convection}

Convection is the process in which heat energy is transferred from place to place by the bulk movement of a fluid. A good example of this process is convection current in liquid.

$\mathrm{J} /\left(\mathrm{s} . \mathrm{m} . \mathrm{C}^{\mathrm{o}}\right)$

By convection, energy loss rate depends on whether the air is forced to flow (e.g. by a fan) or moves by natural convection.

By convection, energy loss rate $=\mathbf{h} \mathbf{A}\left(\mathbf{T}_{\mathbf{s}}-\mathbf{T}_{\mathbf{0}}\right)$

\subsubsection{Radiation}

This is a process in which energy is transferred by means of electromagnetic waves. A good example of this is the solar radiation from the sun traveling in all directions in space. Part of this radiation is reaching the earth on daily basis and in actual sense; the bulk of energy on earth is from the sun. All bodies, hot or cold, continuously radiate energy in form of electromagnetic waves. But the amount of this radiation is proportional to the temperature of the body and the nature of its surface.

By radiation, energy loss rate $=\sigma \mathbf{A}\left(\mathbf{T}_{\mathbf{o}}{ }^{4}-\mathbf{T}_{\mathbf{s}}{ }^{4}\right)$

Stefan-Boltzmann Law of Radiation

This law state that all matter constantly radiates energy in form of electromagnetic waves.

$$
Q / t=\sigma \varepsilon A T^{4}
$$

where $\sigma$ is the Stefan-Boltzmann constant, $\varepsilon$ is emissivity and it has

value between 0 and 1 . For a perfect reflector, $\varepsilon=0$ and for a black

body e $=1$.

Equation 4 is known as the Stefan-Boltzmann law of radiation, and the law stated that the radiant energy, emitted in a time $\mathbf{t}$ by an object that has a Kelvin temperature of $\mathbf{T}$, a surface area A, and an emissivity e, is given by $Q=\varepsilon \sigma T^{4} A t$.

Emissivity $\varepsilon$ of an Object: This is the ratio of radiant energy emitted by an object to the one it would have emitted if it were to be a perfect black body (Halliday etal, 2001).

$\varepsilon=\frac{\text { radiation emitted }}{\text { RadPbb }}$

\subsubsection{Conduction}

This is the process of heat transfer whereby heat energy is transferred directly through a material without any bulk movement of the material. 
Consider a bar of material of area A and thickness L, heat Q transfer through the material by conduction is

$$
\mathrm{Q}=\frac{\mathrm{KATt}}{\mathrm{L}}
$$

where $\square \boldsymbol{T}$ is the temperature difference between the ends of the bar and $\mathrm{k}$ is the thermal conductivity of the material. Unit of $\mathrm{k}$ is $\mathrm{J} /\left(\mathrm{s} \cdot \mathrm{m} . \mathrm{C}^{\mathrm{o}}\right)$

\section{Conduction of Heat through a Composite Material}

Consider two materials of thickness $\boldsymbol{L}_{\mathbf{1}}$ and $\boldsymbol{L}_{\mathbf{2}}$ with different thermal conductivities $\boldsymbol{k}_{\mathbf{1}}$ and $\boldsymbol{k}_{\mathbf{2}}$ respectively as shown in figure 4.1. The outer surfaces of the slab are in thermal contact with hot reservoir at temperature $\boldsymbol{T}_{\boldsymbol{H}}$ and cold reservoir at temperature $\boldsymbol{T}_{\boldsymbol{C}}$. Assuming that the heat transfer through the slabs is a steady rate process i.e. the temperature everywhere in the slab and the rate of energy transfer do not change with time. Then, the conduction rate through the two slabs must be equal. Using equation 4.1, the conduction rate $P_{\text {cond }}$ is

$P_{\text {cond }}=\frac{\mathrm{Q}}{\mathrm{t}}=\frac{\mathrm{KA} \Delta T}{\mathrm{~L}}$

Let $\boldsymbol{T}_{\boldsymbol{X}}$ be the temperature of the interface between the two materials, so

$$
P_{\text {cond }}=\mathrm{k}_{2} \mathrm{~A} \frac{(T H-T X)}{\mathrm{L} 2}=\frac{\mathrm{K} 1 \mathrm{~A}(T X-T C)}{\mathrm{L} 1}
$$

Solving for TX in equation [8] gives

$\mathrm{TX}=\frac{\mathrm{K} 1 \mathrm{~L} 2 \mathrm{TC}+\mathrm{K} 2 \mathrm{~L} 1 \mathrm{TH}}{\mathrm{K} 1 \mathrm{~L} 2+\mathrm{K} 2 \mathrm{~L} 1 \mathrm{TH}}$

Insert equation [9] in equation [8] to obtain

$$
P_{\text {cond }}=\frac{A\left(T_{H}-T_{X}\right)}{L / \mathbf{K} \mathbf{1}-\mathbf{L} \mathbf{2} / \mathbf{K} \mathbf{2}}
$$

If we apply this to any number $\mathrm{n}$ of materials, equation [10] becomes

$$
n \quad P_{\text {cond }}=\frac{A(T H-T X) \text { for } i}{\sum 2^{1}\left(L / i_{k i}\right)}=2: n
$$

\subsection{QUANTUM RADIATION}

Radiation is the process by which heat is conveyed from one place to another without heating the intervening medium. Radiant energy from the sun travels for most of the way through empty space and this energy displays electrical and magnetic properties and is said to be electromagnetic radiation (Okoye and Maduka, 2015). Under the intense sunlight, the electrical and magnetic properties from the sun shines on aluminum ladder placed five meters gap parallel to $\mathrm{G}$ and $\mathrm{P}$ unit of $100 \mathrm{Kva}$ transformer accessories drawn from $33 \mathrm{Kva}$ line, the electron on the aluminum ladder would be powered by this radiant energy thereby generating current on its own whereas the transformer is $\mathrm{ON}$ and not isolated. In practical, this process describe is very dangerous because two different sources of light is flowing spontaneously on the aluminum ladder and on the transformer respectively. When the two current flows in the same direction toward each other, they act as a magnet causing the aluminum ladder to be attracted by the electrical force on the $\mathrm{G}$ and $\mathrm{P}$ unit of the transformer since the heavier amount of current is flowing on the transformer compare to the few voltage of current generated on the aluminum ladder by the radiant energy. Force of attraction acts on two parallel conductors carrying current in the same direction. The collision of this two conductors carrying current is always deadly to the operator who is trying to set the aluminum ladder before isolating the transformer from the grid or may be because the operator believed that he placed the aluminum ladder 5 meters gap from the $\mathrm{G}$ and $\mathrm{P}$ unit of the transformer. This radiation travels with the speed of light, about $3 \times 10^{8}$ $\mathrm{m} / \mathrm{s}$. Radiant energy is emitted (or absorbed) in BURSTS i.e. in fixed amounts called Quanta. There are instances where light behaves as if it were composed of tiny packets or Quanta of energy called Photons. The energy, E, of the photon emitted or absorbed by substances i.e. the energy of one quantum of radiation is proportional to the frequency of the light

$\mathrm{E}=\mathrm{hv} . .$.

Where

$\mathrm{E}=$ photons or quantum of energy

$\mathrm{h}=$ planck's constant

$\mathrm{v}=$ frequency. 
The quantum energy is also related to the wavelength of the radiation:

$\mathrm{E}=\mathrm{hC} / \lambda$

Where

$\mathrm{C}=$ velocity of light

$\lambda=$ wavelength of the radiation

$\mathrm{E}=$ Photon of quantum of energy

$\mathrm{h}=$ Planck's constant $\left(6.63 \times 10^{27} \mathrm{erg}\right.$ Sec. $)$

\subsection{THERMAL RADIATION PROPERTIES}

The continuous power rating of a resistor, $P_{R}$, is based on three factors.

(1) Maximum surface temperature, in free air, over the usable ambient temperature range, typically from $-55^{\circ} \mathrm{c}$ to well over $100^{\circ} \mathrm{c}$.

(2) Stability of resistance when subjected to a dc cyclic load. Typical power dissipation is limited to give 5 percent resistance change for 2000 hours continuous operation in a $70^{\circ} \mathrm{c}$ ambient air temperature.

(3) Proximity of other heat sources and the flow of cooling air.

The temperature rise of a resistor due to power dissipation is determined by the laws of conduction, convection and radiation (Williams,1992). The maximum body temperature, the hot spot temperature, occurs at the nominal operating temperature range of a resistor, the hot spot temperature, $T_{h}$, is given by

$\mathrm{T}_{\mathrm{h}}=\mathrm{T}_{\mathrm{a}}+\mathrm{R}_{\theta \mathrm{h}-\mathrm{a}} \mathrm{P}_{\mathrm{d}}(\mathrm{K})$.

Where

$\mathrm{T}_{\mathrm{a}}$ is the ambient Temperature

$\mathrm{T}_{\mathrm{h}}$ is the hot spot temperature.

The hot temperature is limited thus as the ambient temperature, $\mathrm{T}_{\mathrm{a}}$, increases the allowable power dissipated decreases.

\subsection{PROPERTIES OF SEMICONDUCTORS}

A semiconductor is a material with electrical properties partway between those of a conductor and an insulator. The outermost electrons in a conductor (e.g Metal) are free to move in the material whereas an insulator cannot move in the same way, so they cannot carry current. In a semiconductor, only a few of the electrons are free to move in the material. The major difference among conductors, semiconductors and insulators is in how the electrons are bound to atoms in the material.

Insulators are materials that hold onto their electrons tightly, because the outer electrons form bonds between atoms.

Conductors are materials in which the outer electrons are loosely bound to atoms; metallic aluminum, with three electrons in its outer shell, is an example. In semiconductors such as pure silicon, most electrons are bound to the crystalline lattice, but a few can escape to travel within the material.

The electrons in semiconductors can fall into the two energy bands; valence band and conduction band (Okoye and Maduka, 2015). The number of electrons in each band depends on the size of the energy difference or " band gap" between conduction and valence bands, and on the Boltzmann law;

$\mathrm{N}_{2} / \mathrm{N}_{1}=\exp \left[-\left(\mathrm{E}_{2}-\mathrm{E}_{1}\right) / \mathrm{KT}\right]$

Where $\mathrm{N}_{2}$ and $\mathrm{N}_{1}$ are the numbers of electrons in the conduction and valence band, respectively, $\mathrm{E}_{2}-\mathrm{E}_{1}$ is the energy difference between the two states (the band gap), $\mathrm{K}$ is the Boltzmann constant, and $\mathrm{T}$ is the temperature (in kelvin or absolute). The energy required for the transition from a state of lower energy $\left(E_{1}\right)$ to state of higher energy $\left(E_{2}\right)$ is exactly equivalent to the energy of electromagnetic radiation that causes transition.

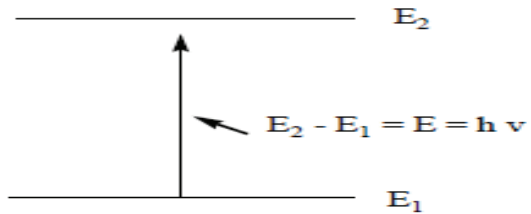

Fig.2.0: Energy transition for the absorption of any electromagnetic radiation

Note that the Band gap of Trichlorosilane molecules could be obtained from the Fig.2.0

$$
\begin{aligned}
& \mathrm{E}=\mathrm{E}_{2-} \mathrm{E}_{1=} \mathrm{hu} \\
& \mathrm{E}=\mathrm{h} \frac{\mathrm{c}}{\lambda} \ldots \ldots \ldots
\end{aligned}
$$


Where $\mathrm{E}$ is photon energy of electromagnetic radiation being absorbed in $(\mathrm{eV}), \mathrm{h}$ is the universal Planck's constant, $6.624 \times 10_{8}^{-34} \mathrm{Js}$ and $\mathrm{v}$ is the frequency of incident light in cycles per second (cps or hertz, Hz), c is velocity of light $3.0 \times 10^{8} \mathrm{~m} /$ sand $\lambda=$ wavelength $(\mathrm{nm})$.

Therefore, as we move from cosmic radiations to ultraviolet region to infrared region and then radio frequencies, we are gradually moving to regions of lower energies.

A molecule can only absorb a particular frequency, if there exists within the molecule an energy transition of magnitude $\mathrm{E}=$ hv

Although almost all parts of electromagnetic spectrum are used for understanding the matter, in organic chemistry we are mainly concerned with energy absorption from only ultraviolet and visible, infrared, microwave and radiofrequency regions.

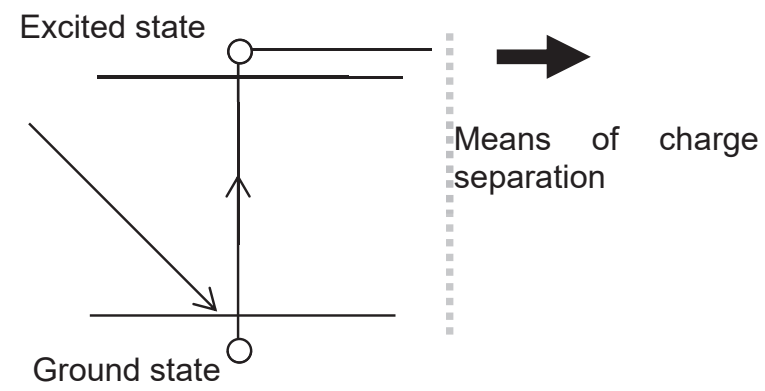

Fig. 3.0: Excitation and charge separation.

\subsection{THERMAL RESISTANCES}

Semiconductor power losses are dissipated in the form of heat, which must be transferred away from the switching junction (Williams, 1992). The reliability and life expectancy of any power semiconductor are directly related to the maximum device junction temperature experienced. It is therefore essential that the thermal design determines accurately the maximum junction temperature from the device power dissipation.

The basic equation for heat transfer under steady state conditions.

$\mathrm{P}_{\mathrm{d}}=\mathrm{h} \mathrm{A} \Delta \mathrm{T}(\mathrm{W})$

Where

$\mathrm{P}_{\mathrm{d}}$ is the rate of heat transfer

$\mathrm{h}$ is the heat transfer coefficient

A is the area involved in the heat transfer

$\Delta \mathrm{T}$ is the temperature difference between regions of heat transfer.

\subsection{FORCE OF ATTRACTION BETWEEN A CURRENT CARRYING CONDUCTORS}

In a magnetic field, a current carrying conductors exert a force on each other ( Otuka et al, 2014). The direction of current flow in each wire determines whether the force acting between the conductors would be attractive or repulsive (Anyakoha, 2011 ). If the current in the two long neighbouring straight conductors is flowing in the same direction, the force between two such conductors is an attractive force, causing the two conductors to move towards each other. If the current flows in opposite direction in the two conductors, the force between the conductors is a repulsive force. For the purpose of this research, assuming that the current on the two specimens are flowing in the same direction there is going to be an explosion of fire and electrocution.

$\mathrm{F}=\mathrm{Ma}$.

\subsection{IMPACT OF PHOTOEMISSION ON THE ALLUMINIUM LADDER \\ 1.9.1 PHOTOCHEMICAL REACTION}

The energy carried in a light wave or photon can trigger chemical reaction on aluminum ladder because a photon causes a chemical change (Jeff,1993). Light energy can break apart a complex molecule, remove an atom or molecule or excite an atom or molecule into a state where it is more ready to engage in chemical reactions.

Ordinary light, from the sun or a light bulb, is emitted spontaneously, when atoms or molecules release excess energy by themselves, without any outside intervention. Stimulated emission is different, because it occurs when an atom or molecule that is holding excess energy is "stimulated" to emit that energy as light. 


\subsection{IMPACT OF MAGNETIC RADIATION BETWEEN CURRENT CARRYING CONDUCTORS IN THE SAME DIRECTION IN A MAGNETIC FIELD.}

In a magnetic field, two parallel conductors carrying current moving in the same direction separated within the distance below 5 meters will attract each other. Each wire will exert a force on each other if the distance is not upto 5 meters and if the current flow in the same direction, force of attraction will automatically exist between these wires attracting them to each other. In real practical, force of attraction between current carrying conductors in the field always collide without the knowledge of the technician.

This is why it is very deadly to set aluminum ladder side by side very close to transformer gantries ( $\mathrm{G}$ and $\mathrm{P}$ units) without isolating the transformer source from the grid, especially when the distance of separation between the ladder and the transformer gantries unit is between 5 meters or below 5 meters range. Whenever there is magnetic attraction, it is always deadly; there will be a big force of explosion resulting to burning and cutting of many accessories in transformers; feeder pillar unit, G and P unit and network. This attraction can equally leads to electrocution if there is an operator working on this aluminum ladder without first of all isolating the transformers current from the gridline. This type of disaster has resulted to the death of many careless technicians while many lucky once escape the unfriendly incidence. The impact of magnetic attraction of current carrying conductors is not good to experience in practical because it always lead to breakdown of the transformer accessories, feeder pillar, grid line, and sometimes lost of life.

\subsection{MITIGATING THE DANGERS OF MAGNETIC ATTRACTION OF CURRENT CARRYING CONDUCTORS IN THE SAME DIRECTION IN A MAGNETIC FIELD}

We have to put the following measures in place in order to avoid or reduce this type of disasters in our field;

1. The technicians should be train to acquire the necessaries knowledge and skill required for them to operate fervently on the transformer and the grid unit of electricity network.

2. The technician should always ensure that they isolate the supply coming to the transformer from the grid before working on the transformer unit.

3. The technician should ensure that they take outage whenever they want to work on the grid line.

4. The technician should ensure that they disconnect the transformer fuse and transformer unit on the feeder pillar whenever they want to work on the low tension line.

5. The technician should also endevour to wear protective kits all over their body before embarking on any maintenance or reinforcement work on the grid.

6. The technician should always ensure that they observe distance of more than 5 meters away before placing another conductor carrying current to avoid force of attraction as a result of thermal radiation from heated electrical part or conduction as a result of excited energy from the sun (Photon).

7. The technician should ensure that the isolator unit on the transformer or R.P.U unit on the grid network is functioning properly before depending on the ability of this unit.

8. The technicians should ensure that aluminum ladder or any metal object they used as their working tools are coated right round with plastic material or insulated with insulating material of higher efficiency.

\subsection{CONCLUSION}

Heat can be transferred in or out of a system by one or more of these three mechanism; conduction, convection and radiation. Thermal radiation occurs within the circumference of any gantry that is carrying transformer accessories connected to a source of electricity. The thermal radiation can occur in any of the following means; conduction, convection and radiation. This research reveals that when thermal radiation took place within the transformer gantry carrying two conductors supplying current in the same direction, magnetic force of attraction can take place doing the peak period of sunlight when the number of photon energy transferred by the sunlight is equal to the number of electron existing in the surface of the two conductors despite the 5 meters separation distance existing within the two arms of the conductors. The operators or technicians of power network should have this phenomenon in mind that electric current generated through solar energy is real and can cause two conductors carrying electric current from a source to act as a magnet even when there is 5 meters distance separation existing within them. This happened because natural current from solar energy always generated and transmitted doing the intense sunlight by the day. And again, the gantry when heated up by a source can equally generate thermal radiation which is capable of causing magnetic influence within the two conductors carrying 
current in the same direction. The best safety tips is to off or isolate current supply from reaching any unit which technicians want to work on before setting the aluminum ladder or before climbing the pole to carry out any form of work on the network. Conductors conduct Charges in terms of thermal conductivity because of collision mechanism and free electrons movement that exist in metals.

Molecular collision: Atoms and molecules in a hot part of the material vibrate or move with greater velocity (i.e. higher kinetic energy) than those at the colder part. By means of collisions, the more energetic molecules pass on a portion of their energy to their less energetic neighbours. As the more energetic molecules collide with their less energetic neighbours they transfer some of their energy to the neighbours. The collision mechanism does not depend on bulk movement of the material.

Good conductors of thermal energy conduct energy because of the existence of pool of electrons that are more or less free to wander through the volume of the metal. These free electrons are capable of transporting energy round the whole volume of conductors. Free electrons are also responsible for the excellent electrical conductivity in metals.

Materials that conduct heat poorly are called thermal insulators. These materials poorly conduct heat energy because the two above discussed mechanisms for conduction are not possible with these materials (i.e. the molecules of these materials are not free to move and or the material don't contain free electrons).

\section{REFERENCES}

Adams, Steve and Halliday, Jonathan (2000). Advanced Physics.UK: Oxford University Press.

Francis, W. Sears and Gerhard, L. Salinger (1975). Thermodynamics, Kinetic Theory, and Statistical Thermodynamics (Third Edition). Philippines: Addison-Wesley Publishing Company, Inc.

Halliday, David; Resnick, Robert and Walker, Jeanrl (2001). Fundamentals of Physics-Extended (Sixth edition). New York: John Wiley and Sons, Inc.

John, D. Cutnell and Kenneth, W. Johnson (1989). Physics. USA: John Wiley \& Sons, Inc.

Mark, W. Zemansky and Richard, H. Dittmann (1981). Heat and Thermodynamics (Sixth Edition). New York: McGraw-Hill Book Company.

Okoye,I.F and Maduka, N.C (2017). The investigations of an efficient multi-crystal silicon solar cell. Journal of Nigerian Association of Mathematical Physics Volume 41,(May,2017),pp423-43. ISSN:1116-4336.

Okoye,I.F (2017). Basic Applications in Energy and Power. Text Book publication of Ahmadu Bello University Press Limited, Publisher and Printers. ISBN 978-978-853624-6

Stuart, B. Palmer and Mircea, S. Rogalski (1996). Advance University Physics. SA: Gordon and Breach Science Publisher.

Rao, Y.V.C. (2004). An Introduction to Thermodynamics (Revised Edition). India: University Press (India) Private Limited.

G.Anene and A.J Ekpunobi(1998). University Physics, Modern Physics and Relativity (For Science and Engineering Students). Nigeria: Gospel Press, Enugu-Nigeria.

Jeff, Hecht. (1992). Understanding Lasers An entry-level Guide (Second Edition). New York: IEEE Press Understanding Series.

B.W. Williams.(1992). Power Electronics Devices, Drivers, Applications and Passive Components (Second Edition). Hong Kong: MACMILLAN PRESS LTD, Houndmills, Basingstoke, Hampshire RG21 2 X S and London.

M.W. ANYAKOHA. (2011). New school Physics (Fourth Edition). Nigeria: Africana First Publishers Plc, Onitsha,Nigeria.

J.O.E.Otuka, E.O.Alao and E.I.Pati. (2014). Exam Focus Physics. Nigeria: University Press Plc, Ibadan.

\footnotetext{
About the author: Mr. Okoye Ikechukwu Francis, is a holder of M.Tech, B.Tech in Physics/Electronics from Nnamdi Azikiwe University, Awka, Anambra State Nigeria. Okoye Ikechukwu Francis is also a Ph.D student of Pure and Industrial Physics in Solar Energy in the same university. Mr. Okoye Ikechukwu Francis is currently a Permanent Lecturer in Physics Department of Federal University, Gusau, Zamfara State Nigeria. Okoye Ikechukwu Francis, have Author and Co-author text books and journals in special areas of Physics and applied physics. Okoye Ikechukwu Francis is formally the Examination Officer of Physics Department and currently the coordinator of Student Industrial Work Experience Scheme(SIWES) in the same department. Mr. Okoye Ikechukwu Francis is Married with Kids.
} 